\title{
Cash transfer narratives and charitable giving
}

\author{
Justin Abraham ${ }^{* \ddagger \ddagger}$, Nicholas Otis ${ }^{\ddagger \ddagger}$, Catherine Thomas ${ }^{\ddagger \ddagger}$, Hazel Markus ${ }^{\ddagger}$, Gregory Walton ${ }^{\ddagger}$
}

February 23, 2019

\section{Contents}

1 Introduction 2

2 Research Design 2

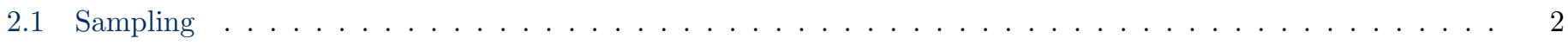

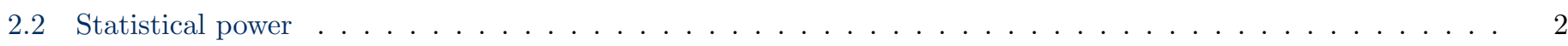

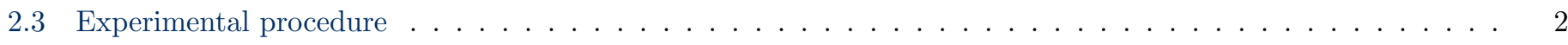

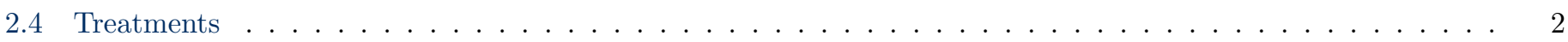

3 Data 3

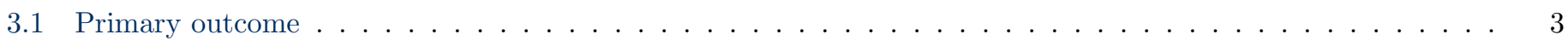

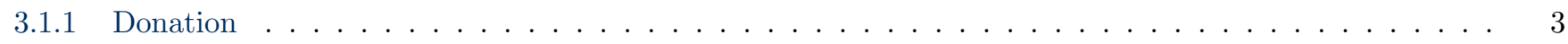

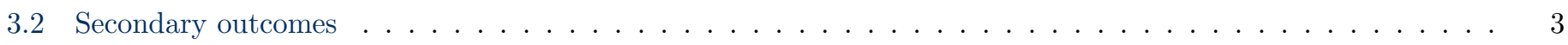

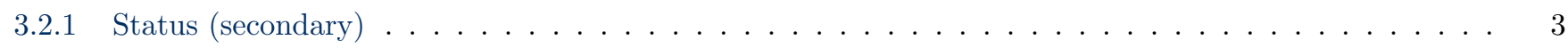

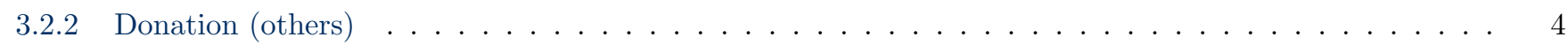

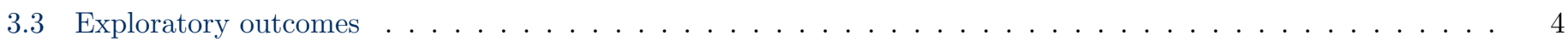

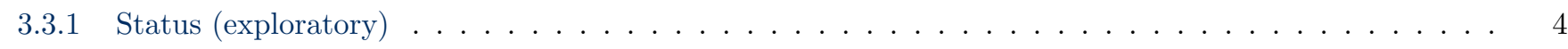

3.3.2 Construals of recipient need . . . . . . . . . . . . . . . . . . . . . . . 4

3.4 Comprehension check module . . . . . . . . . . . . . . . . . . . . . . . . . 5

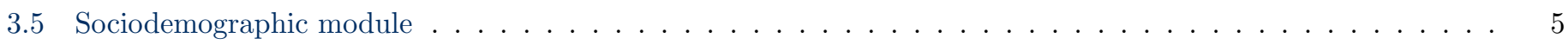

4 Empirical Analysis 5

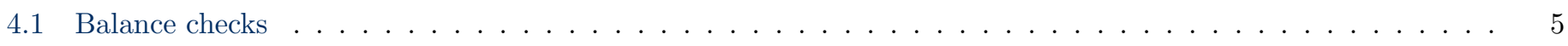

4.2 Treatment effect of cash transfer messages . . . . . . . . . . . . . . . . . . . . . . . . 5

4.3 Covariate adjustment . . . . . . . . . . . . . . . . . . . . . . . . 6

4.4 Heterogeneous treatment effects . . . . . . . . . . . . . . . . . . . . . . . 6

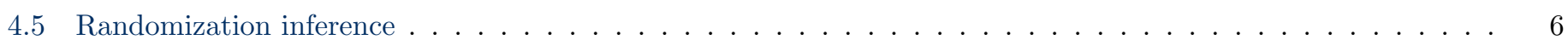

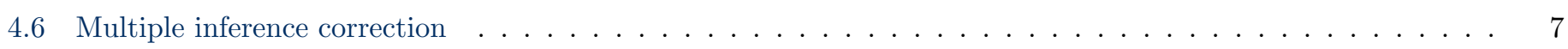

${ }^{*}$ University of California, San Diego. ${ }^{\dagger \dagger}$ Contributed equally.

${ }^{\dagger}$ University of California, Berkeley. ${ }^{\dagger \dagger}$ Contributed equally.

${ }^{\ddagger}$ Stanford University. ${ }^{\dagger \dagger}$ Contributed equally. 


\section{Introduction}

In a previous pre-registered study (Abraham et al., 2017), we examined the effects of narratives accompanying unconditional cash transfers on self-concept and economic behavior through a lab-in-the-field experiment with low income participants in Nairobi, Kenya. We randomized whether participants were told the cash was intended for 1) poverty alleviation, 2) individual empowerment, or 3) community empowerment. To complement our study on recipient behavior, we now explore donor behavior among the same frames we used in Kenya. Participants are asked to read a short description of a charitable organization from our previous study. If donor support is lowest for the worst performing treatments in our Kenyan study, this adds strength to the effectiveness of the frame for both eliciting support and impacting recipient behavior. If donors prefer treatments that perform more poorly among recipients, it presents a policy paradox, whereby frames that maximize donation have deleterious effects on the respondents they were intended to help.

\section{Research Design}

\subsection{Sampling}

We will conduct our experiment on the Mechanical Turk platform. This platform is now commonly used by researchers to conduct online experiments as it provides an inexpensive and efficient way of recruiting respondents that have been shown to respond to experiments similarly to nationally representative sample in the U.S. (Mullinix et al., 2015). We will recruit workers on MTurk over the age of 18 and residing in the United States. We restrict our sample to Mechanical Turkers who have an approval rating above $95 \%$, who have completed more than 50 tasks, and who have not taken a pilot survey with us previously. We will also exclude MTurkers outside the US, who they cannot commit to carefully reading about the nonprofit, who fail both of our basic comprehension questions, or multiple responses from the same IP address. We also exclude people who did not complete a response for our primary outcome. Respondents are compensated $\$ 0.60$ for the five minute survey and are entered into a $\$ 100$ lottery with chances 1 in 200 .

\subsection{Statistical power}

To achieve power of $80 \%$ for an estimated effect size of 0.20 SD on our primary analyses, the required sample size is 1,182 participants, with 394 in each of the treatment arms. Estimating that our comprehension checks will exclude $20 \%$ of participants, we will recruit $1,478=1,182 / 0.8$ participants.

\subsection{Experimental procedure}

\subsection{Treatments}

Each respondent is asked to read about a nonprofit organization operating in Nairobi, Kenya, East Africa. We randomly assign the description of the nonprofit organization:

Poverty Alleviation Organization message: "The goal of the Poverty Alleviation Organization is to alleviate poverty and reduce financial hardship among the poor. This organization believes that people living in poverty should be given income support to help them meet their basic needs. This organization aims to help promote a decent standard of living among the poor and help them deal with emergencies. Thus, the Poverty Alleviation Organization gives financial assistance to people to help them make ends meet. For example, with the financial assistance, people might be able to struggle less to afford basic needs, like paying off debts, paying rent, and buying clothes and food.

Towards that mission, this nonprofit gives one-time transfers of financial assistance to people in urban slums in Nairobi, Kenya, East Africa. They give each recipient \$4 USD (about two days wages) in cash. These program recipients are people who live on less than \$2 per day, and half of recipients have no savings."

Individual Empowerment Organization message: "The goal of the Individual Empowerment Organization is to promote individuals' potential to create a better future for themselves. The organization believes that individuals are wise and know best how to help themselves become self-reliant if they have the financial resources to do so. This organization aims to empower 
individuals to pursue their personal interests and create their own path to independence. Thus, the Individual Empowerment Organization gives financial resources to individuals to enable them to invest in their personal goals. For example, people might use their unique talents to start a self-run business, invest in job training courses, or create art.

Towards that mission, this nonprofit gives one-time transfers of financial resources to people in urban slums in Nairobi, Kenya, East Africa. They give each recipient $\$ 4$ USD (about two days wages) in cash. These program recipients are people who live on less than \$2 per day, and half of recipients have no savings."

Community Empowerment Organization message: "The goal of the Community Empowerment Organization is to enable people to help promote better futures for those they care about and want to support most. The organization believes that people know best how to support each other and grow together if they have financial resources to do so. This organization aims to empower people to improve their own lives and those of the people and communities they care about most. Thus, the Community Empowerment Organization gives financial resources to community members to enable them to contribute positively to the lives of people important to them. For example, when people can invest in themselves, they are better able to expand employment opportunities for others, provide valuable services to their community, or teach others, including children, useful skills and knowledge.

Towards that mission, this nonprofit gives one-time transfers of financial resources to people in urban slums in Nairobi, Kenya, East Africa. They give each recipient $\$ 4$ USD (about two days wages) in cash. These program recipients are people who live on less than \$2 per day, and half of recipients have no savings."

\section{Data}

This section describes the data collected following the the treatments described above. These measures are collected in the order listed below. We have only one primary outcome (amount donated). All other outcomes are considered secondary or exploratory.

\subsection{Primary outcome}

\subsubsection{Donation}

Our primary outcome is a behavioral measure of the respondents willingness to donate to a charity (relative to keeping the money for themselves). In a secondary analysis, we will analyze this outcome as the a proportion of participants donating by condition. The question states: "As a result of participating in this study, you may also receive a bonus of $\$ 100$. One in two hundred participants will be randomly selected for this bonus. We are giving people the opportunity to donate, in advance, part of that $\$ 100$ to the [ORGANIZATION NAME]. Their mission statement is copied below for your reference. If you are selected for the \$100, how much would you like to donate to this organization versus keep for yourself? (Note the amounts must sum to 100)."

\subsection{Secondary outcomes}

\subsubsection{Status (secondary)}

Ladder (Self): "Think of this ladder as representing where people stand globally. At the top of the ladder are the people who are the best off globally - those who have the most money, the most education and the most respected jobs. At the bottom of the ladder are the people who are the worst off globally - those who have the least money, least education, and the least respected jobs or no job. The higher up you are on this ladder, the closer you are to the people at the very top; the lower you are, the closer you are to people at the very bottom? Where would you place yourself on this ladder? Use the slider to indicate where on the ladder you think you stand at this time in your life, relative to other people in the world." 


\subsubsection{Donation (others)}

To what extent would you be likely to encourage your friends to donate to the [Organization name]? (1=Not at all likely to encourage to $5=$ Extremely likely to encourage)

\subsection{Exploratory outcomes}

\subsubsection{Status (exploratory)}

1. Size of self vs others: Please write the number of the diagram (1 -7) that best represents how you see yourself "Me" compared to others "O"

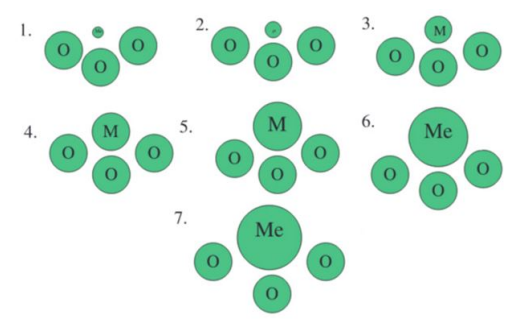

2. Size of self vs. friends:

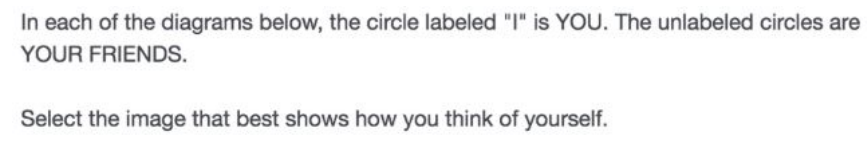

Select the image that best shows how you think of yourself.

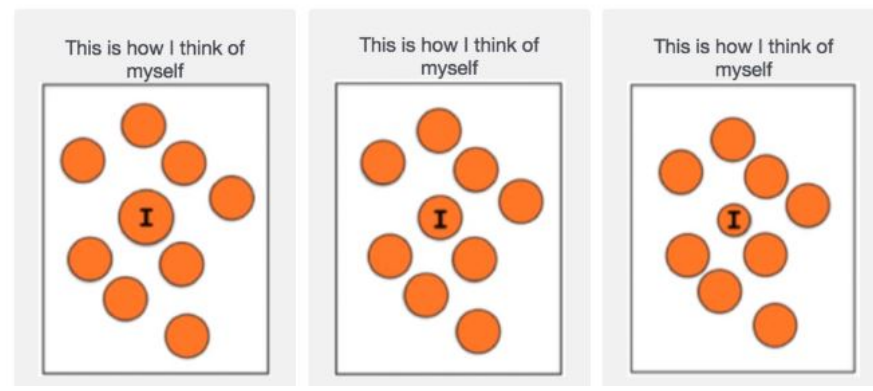

3. Attribution of poverty: Why, in your opinion, are there people in this country who live in need? Here are two opinions: which comes closest to your view? They are poor because of laziness and lack of willpower, or They are poor because society treats them unfairly.

\subsubsection{Construals of recipient need}

1. To what extent will \$4 improve the life of a recipient of the [organization name]?

(1 An enormous amount; 2 A great deal; 3 A lot; 4 A moderate amount; 5 Somewhat; 6 Very little; 7 Not at all)

2. To what extent do you think that recipients of the [organization name] need financial assistance from others?

(1 An enormous amount 2 A great deal; 3 A lot; 4 A moderate amount; 5 Somewhat; 6 Very little; 7 Not at all)

3. Ladder (Recipient): Think of this ladder as representing where people stand globally. At the top of the ladder are the people who are the best off globally - those who have the most money, the most education and the most respected jobs. At the bottom of the ladder are the people who are the worst off globally - those who have the least money, least education, and the least respected jobs or no job. The higher up you are on this ladder, the closer you are to the people at the very top; the lower you are, the closer you are to people at the very bottom. Where would you place recipients of the

[organization name] on this ladder? Use the slider to indicate where on the ladder you think you stand at this time in your life, relative to other people in the world. 


\subsection{Comprehension check module}

1. Please mark how much recipients of this program earn per day, based on the program description. (1=Less than $\$ 2$ per day to $6=$ More than $\$ 10$ per day)

2. Who was described as the recipients of the nonprofit program?

- Mothers in rural areas of Kenya

- Farmers in rural areas of Kenya

- Young men in urban slums in Kenya

- People in urban slums in Kenya

- Elderly people in rural areas of Kenya

\subsection{Sociodemographic module}

1) Have donated to an international aid organization in the past

2) Age

3) Gender

4) Annual household income

5) Number of people in the household

6) Education

7) Race/ethnicity

8) Political affiliation

9) Religious identification

10) Religiosity

\section{Empirical Analysis}

\subsection{Balance checks}

We test for differences in sociodemographic characteristics (imbalance) using the following specification:

$$
Y_{i}=\beta_{0}+\beta_{1} \operatorname{IND}_{i}+\beta_{2} \operatorname{CoM}_{i}+\varepsilon_{i}
$$

where $Y_{i}$ refers to the sociodemographic variables listed in Section 3.5 for individual $i$ measured at the end of the survey. $\mathrm{IND}_{i}$ indicates assignment to the individual empowerment message while $\mathrm{CoM}_{i}$ indicates assignment to the community empowerment message. The reference category in this model is the poverty alleviation message. $\varepsilon_{i}$ is the idiosyncratic error term. Given that our randomization takes place at the individual level, we employ heteroskedasticity consistent robust standard errors here and in subsequent specifications. When estimating treatment effects, we include sociodemographic variables for which we reject balance as control variables.

\subsection{Treatment effect of cash transfer messages}

We will use the following reduced-form specification to estimate the treatment effect of different messages:

$$
Y_{i}=\beta_{0}+\beta_{1} \operatorname{IND}_{i}+\beta_{2} \mathrm{COM}_{i}+\varepsilon_{i}
$$

where $Y_{i}$ refers to the outcome variables for individual $i$ measured after the manipulation. The variables are as described above. We will also test for equivalence among the individual and community conditions, and if these two conditions are jointly different from the poverty condition. 


\subsection{Covariate adjustment}

To improve precision, we will apply covariate adjustment with a vector of baseline indicators $\mathbf{X}_{i}$. We obtain the covariateadjusted treatment effect estimate by estimating Equation 2 including the demeaned covariate vector $\dot{\mathbf{X}}_{i}=\mathbf{X}_{i}-\overline{\mathbf{X}}_{i}$ as an additive term and as an interaction with the treatment indicator:

$$
Y_{i}=\beta_{0}+\beta_{1} \operatorname{IND}_{i}+\beta_{2} \operatorname{Com}_{i}+\gamma_{0} \dot{\mathbf{X}}_{i}^{\prime}+\gamma_{1} \operatorname{IND}_{i} \dot{\mathbf{X}}_{i}^{\prime}+\gamma_{2} \operatorname{Com}_{i} \dot{\mathbf{X}}_{i}^{\prime}+\varepsilon_{i}
$$

The set of indicators partitions our sample so that our estimate for $\beta_{j}$ remains unbiased for the average treatment effect Lin et al. (2013). We use this model to test the hypotheses listed in Section 4.2, including the control variables listed in Section 3.5. Equation 2 without covariate adjustment remains our preferred specification and report both estimates for robustness.

\subsection{Heterogeneous treatment effects}

We will analyze the extent to which the treatments produced heterogeneous treatment effects with the following specification:

$$
Y_{i}=\beta_{0}+\beta_{1} \operatorname{IND}_{i}+\beta_{2} \operatorname{Com}_{i}+\delta_{0} x_{i}+\delta_{1}\left(\operatorname{IND}_{i} \times x_{i}\right)+\delta_{2}\left(\mathrm{Com}_{i} \times x_{i}\right)+\varepsilon_{i}
$$

where $x_{i}$ is the dimension of heterogeneity. $\delta_{1}$ and $\delta_{2}$ identify the heterogeneous treatment effects of the individual empowerment and community empowerment messages relative to the poverty alleviation message. Testing $\delta_{1}=\delta_{2}$ identifies heterogeneous effects between the former two messages. We estimate this model with the variables summarized in Table 1. Because these variables were measured after treatment, we exclude any found to be significantly correlated with treatment. We examine heterogeneous treatment effects for our primary and secondary outcomes.

Table 1: Dimensions of heterogeneity

\begin{tabular}{ll}
\hline Variable & Description \\
\hline Gender & Participant is female \\
Social class & Participants parent has at least a college degree (Stephens et al., 2007) \\
Income & Median split of sample on income (reported annual household income/(no. people in the household $\frac{1}{2}$ ) \\
Donor & Given to an international aid organization before \\
Religiosity & More than slightly religious \\
Party & Party affiliation is Democrat \\
\hline
\end{tabular}

\subsection{Randomization inference}

One potential concern is that inference might be invalidated by finite sample bias in estimates of the standard errors. To address this issue, we will conduct randomization inference to test the Fisherian sharp null hypothesis of no treatment effect for every participant (Fisher, 1935). ${ }^{1}$ We perform Monte Carlo approximations of the exact $p$-values using 10,000 permutations of the treatment assignment. We will then estimate the treatment effect within each $m^{\text {th }}$ permutation and calculate the standard Wald statistics for each of our hypothesis tests. We will compare the Wald statistics from the original sample with the distribution of permuted statistics to produce approximations of the exact $p$-values:

$$
\hat{p}_{\beta}=\frac{1}{10,000} \sum_{m=1}^{10,000} \mathbf{1}\left[\hat{\beta}_{m}^{\prime} V\left(\hat{\beta}_{m}\right)^{-1} \hat{\beta}_{m} \geq \hat{\beta}_{o b s .}^{\prime} V\left(\hat{\beta}_{o b s .}\right)^{-1} \hat{\beta}_{o b s .}\right]
$$

Following (Young, 2015), we will permute the data and calculate the regressions for all outcomes within each draw. We will conduct the permutation test for Equations 2, 3, and 4. While we will highlight analytic $p$-values as primary, we report these bootstrapped $p$-values for robustness.

\footnotetext{
${ }^{1}$ Note that this is more restrictive than the null hypothesis of zero average treatment effect we will test in the previous section.
} 


\subsection{Multiple inference correction}

Since we only have one primary outcome, we will separately calculate sharpened $q$-values over (i) secondary and (ii) exploratory outcomes, and (iii) heterogeneous treatment effects to control the false discovery rate (Benjamini et al., 2006). Rather than specifying a single $q$, we will report the minimum $q$-value at which each hypothesis is rejected (Anderson, 2008). We will apply this correction over each set of outcomes but separately for each hypothesis test and equation. When estimating Equation 4, we correct over different dimensions of heterogeneity separately. We will report standard $p$-values and $q$-values in our analysis. Table 2 summarizes the specified models and methods of statistical inference.

Table 2: Summary of models

\begin{tabular}{lll}
\hline & Treatment effect & Heterogeneous effects \\
\hline Equation 2 & Yes* & Yes* \\
Equation 2 with imbalanced covariates & Yes* & Yes* \\
Equation 3 & Yes* & No \\
\hline
\end{tabular}

*Inference using robust standard errors, approximations of the exact $p$-value, and $p$-values controlling for the FDR. 


\section{References}

Justin Abraham, Nicholas Otis, Catherine Thomas, Hazel Markus, and Greg Walton. Pre analysis plan: Cash Transfer Narratives. 2017.

Michael L Anderson. Multiple inference and gender differences in the effects of early intervention: A reevaluation of the abecedarian, perry preschool, and early training projects. Journal of the American statistical Association, 103(484):1481$1495,2008$.

Yoav Benjamini, Abba M Krieger, and Daniel Yekutieli. Adaptive linear step-up procedures that control the false discovery rate. Biometrika, 93(3):491-507, 2006.

Ronald Aylmer Fisher. The design of experiments. Oliver \& Boyd, 1935.

Winston Lin et al. Agnostic notes on regression adjustments to experimental data: Reexamining freedmans critique. The Annals of Applied Statistics, 7(1):295-318, 2013.

Kevin J Mullinix, Thomas J Leeper, James N Druckman, and Jeremy Freese. The generalizability of survey experiments. Journal of Experimental Political Science, 2(2):109-138, 2015.

Nicole M Stephens, Hazel Rose Markus, and Sarah SM Townsend. Choice as an act of meaning: the case of social class. Journal of personality and social psychology, 93(5):814, 2007.

Alwyn Young. Channeling fisher: Randomization tests and the statistical insignificance of seemingly significant experimental results. E, 0:0-0, 2015. 